\title{
Silencing of a Unique Integrated Domain Nucleotide-Binding Leucine-Rich Repeat Gene in Wheat Abolishes Diuraphis noxia Resistance
}

\author{
Vittorio Nicolis and Eduard Venter ${ }^{\dagger}$ \\ Department of Botany and Plant Biotechnology, University of Johannesburg, Auckland Park, Johannesburg, 2006, South Africa
}

Accepted 7 March 2018.

\begin{abstract}
Plants respond in a similar manner to aphid feeding as to pathogen attack. Diuraphis noxia is a specialist aphid, feeding only on selected grasses that include wheat, barley, and oats. The wheat-Diuraphis noxia interaction is characterized by responses very similar to those seen in wheat-pathogen interactions with none of the underlying resistance pathways and genes characterized yet. From wheat harboring the Dn1 resistance gene, we have identified a nucleotide-binding leucinerich repeat (NLR) gene containing two integrated domains (IDs). These are three $\mathrm{C}$-terminus ankyrin repeat domains and an N-terminus WRKY domain. The NLR core of the gene can be traced through speciation events within the grass family, with a recent WRKY domain integration that is Triticum-specific. Virus-induced gene silencing of the gene in a resistant wheat line resulted in the abolishment of the resistance response and induced a highly susceptible phenotype. Silenced plants supported a higher number of aphids, similar to the susceptible near-isogenic line (NIL), and the intrinsic rate of increase of the aphids matched that of aphids feeding on the susceptible NIL. The presence of the gene is necessary for $\mathrm{DnI}$ resistance and we have named the gene Associated with Dn resistance 1 (Adnrl) to reflect this function.
\end{abstract}

The Russian wheat aphid (Diuraphis noxia Kurdjumov) is a specialist aphid pest of grasses. Its primary hosts with commercial importance are wheat, barley, and oats, while it can survive well on false barley, wild oats, and rescue grass (Jankielsohn 2013). After introduction and escalation into a pest, D. noxia causes tremendous losses in wheat production countries (Morrison and Peairs 1998). Resistant wheat cultivar development resulted in lower yield losses being incurred but, also, increased pressure on the aphids to develop new biotypes. To date 12 resistance genes ( $D n$ genes) have been identified and employed in developing resistant cultivars. Several of these genes were incorporated into resistant wheat lines in South Africa and this has led to the development of at least four known aphid biotypes (RWASA1 to RWASA4) that overcame

${ }^{\dagger}$ Corresponding author: Eduard Venter; E-mail: eduardv@uj.ac.za

Funding: This work was funded by public grants from the Winter Cereal Trust (WCT/W/2007/04) and the National Research Foundation Technology and Human Resources for Industry Programme (TP2009072000010 and TP2011070700029).

*The $\boldsymbol{e}$-Xtra logo stands for "electronic extra" and indicates that one supplementary figure and one supplementary table are published online.

() 2018 The American Phytopathological Society all but the $D n 7$ resistance gene (Jankielsohn 2016). The Dn1 resistance gene, effective against RWASA1, has an antibiotic effect against the aphids and limits their fecundity, growth, and longevity (Smith et al. 1992). This gene was the first gene to be implemented in resistant wheat breeding and maps to chromosome 7DS (Bierman 2015). The gene has yet to be identified or cloned and the mechanism by which it contributes resistance is not clear.

Plants under attack by phloem-feeding insects respond similarly to attack by pathogens (Bos et al. 2010; Rodriguez and Bos 2013). Diuraphis noxia feeding progresses through probing on the leaf surface, intercellular stylet navigation, and, finally, penetration into the phloem to feed. During the intercellular phase, the stylet occasionally pierces cells for the aphid to sense its way. During transition through the leaf, the stylet is protected by the production of a salivary sheath that encloses the stylet. This sheath consists of saliva that solidifies once it is secreted from the stylet tip. Once the stylet reaches the phloem, the aphid starts to produce watery saliva that assists in feeding and that contains effector molecules that interfere with the plant's defense responses (Bos et al. 2010; Lapitan et al. 2007; Rodriguez and Bos 2013; Will et al. 2007). Indeed, it has been shown by Lapitan et al. (2007) that the injection of proteins from different salivary fractions induces symptoms in a susceptible cultivar. This included chlorosis visualized as chlorotic lesions and streaking, leaf rolling, and stunted growth. In contrast, the resistance response is likened to the classic gene-for-gene interaction mediated by a resistance gene with hypersensitive-linked necrotic lesions observed at the site of aphid feeding (Botha et al. 2005).

Nucleotide-binding leucine-rich repeat (NLR) genes often work as dimers to form receptor complexes to specify resistance against a pathogen (Sinapidou et al. 2004). In cereals, several NLR-complex receptors have been identified, with Lr10 and RGA2 the first identified from wheat (Loutre et al. 2009). Studies on rice blast resistance alleles indicated that resistance is imparted by a combination of two genes located at the same locus, e.g., the Pikm and Pia loci (Ashikawa et al. 2008; Okuyama et al. 2011). Thus, interaction between NLRs are indicative of a complex avirulence effector-recognition system that plants employ during innate immunity. Interaction between NLRs to provide resistance can include multiple proteins working in conjunction, as seen in barley resistance against Puccinia graminis. Here, the NLR rpg4 functions together with Rpg5, HvRgal, and $H v A d f 3$, an actin-depolymerizing factor-like gene (Wang et al. 2013). RPG5 contains an additional serine/threonine protein kinase domain that could implicate this NLR in signal transduction. Thus, pathogen recognition by the RPG5/HvRGA1 complex may initialize 
signal transduction by the phosphorylation of serine/threonine protein kinase domains (Wang et al. 2013). This was postulated after the observation that the RPG1 resistance gene in barley contains a similar serine/threonine protein kinase domain that is rapidly phosphorylated in the resistant line against Puccinia graminis $\mathrm{f}$. $\mathrm{sp}$. tritici but not in the susceptible line (Nirmala et al. 2010). This domain could also be targeted by the rust effector and is guarded by the NLRs that are bound to it. Decoy domain recognition, in which the RPS4/RRS1 dimer interacts directly with two effectors, namely PopP2 and AvrRps4, via an integrated WRKY domain to induce a defense response, was demonstrated by Sarris et al. (2015) and Le Roux et al. (2015).

NLR genes that contain WRKY integrated domains (NLRID) at their C-terminus have been identified to act as decoy binding sites for interaction with effector proteins (Le Roux et al. 2015; Sarris et al. 2015). RRS1-R and RPS4 impart resistance to Arabidopsis against Ralstonia solanacearum and Pseudomonas syringae pv. pisi (Sarris et al. 2015). PopP2 is an acetyltransferase that specifically acetylates the lysine residues located in the WRKYQK motif of RRS1 and other nuclear localized WRKY transcription factors that then interfere with their DNA-binding capability, thus disabling transcription activation and subsequent defense responses. Acetylation disrupts the DNA binding of RRS1-R and, in turn, activates RPS4dependent resistance by releasing the RRS1/RPS4 complex inducing innate immunity in Arabidopsis, thus turning the pathogen's effector against itself to induce innate immunity in the cell (Le Roux et al. 2015).

The identification of NLR-ID decoy proteins that dimerize to target effector proteins has furthered our understanding of the complexity of plant innate immunity against pathogens. Identification of a similar NLR-ID protein from wheat that is implicated in the defense response to $D$. noxia has led us to functionally test the role that it plays in the wheat $-D$. noxia interaction. We hypothesized that $5 A L-B 4$, a C-terminal WRKY containing a NLR-ID with additional N-terminal ankyrin repeats, plays a role during the innate immunity of wheat carrying the Dnl resistance gene against $D$. noxia. It is intriguing that $5 A L-B 4$ contains integrated WRKY and ankyrin domains, as ankyrin, WRKY10, WRKY 2, and WRKY53 transcription factors have previously been associated with
D. noxia resistance (Smith et al. 2010; Van Eck et al. 2010). Therefore, the aims were to further characterize $5 A L-B 4$ and to use virus-induced gene silencing (VIGS)-mediated silencing to ascertain the role that $5 A L-B 4$ plays during wheat interacting with D. noxia.

\section{RESULTS}

\section{AL-B4 homologs are genetically similar.}

TRIAE_CS42_5AL_TGACv1_374266_AA1195550 (November 2014 Triticum aestivum Ensemble release; designated $5 A L-B 4$ hereafter) is an NLR with similar architecture to NLRID decoys against pathogen effectors (Fig. 1). It shared homology with Pi36 and was identified as a possible role player in the wheat $-D$. noxia interaction from the study by Nicolis et al. (2017). The sequence and domain architecture for $5 A L-B 4$ was predicted from cultivar Chinese Spring, which has no resistance to $D$. noxia. Polymerase chain reaction (PCR) amplification and sequencing of $5 A L-B 4$ from the $D$. noxia-susceptible nearisogenic line (NIL) Tugela and the resistant NIL Tugela DN was used to confirm the presence of predicted domains and to search for single nucleotide polymorphisms (SNPs) between the resistant and susceptible NILs. No SNPs were identified between the resistant, susceptible, and Chinese Spring cultivars and all predicted domains from Chinese Spring were preserved throughout both NILs. These predicted and confirmed domains included three $\mathrm{N}$-terminal ankyrin repeats followed by a coiledcoil (CC) NLR architecture with a single C-terminal WRKY domain (Fig. 1). The position of the WRKY domain in $5 A L-B 4$ is consistent with the recently postulated NLR-ID genes. This position is further consistent with the recently characterized function of RRS1 that interacts with PopP2 and AvrRps4 (Le Roux et al. 2015; Sarris et al. 2015).

\section{$5 A L-B 4$ is regulated}

\section{during the wheat $-D$. noxia interaction.}

The possible differential regulation of $5 A L-B 4$ in RWASA1-infested resistant NIL Tugela DN and susceptible NIL Tugela was tested using droplet digital PCR (ddPCR). The use of ddPCR for transcript level determination was due to the low levels of NLR expression reported, rendering amplification to
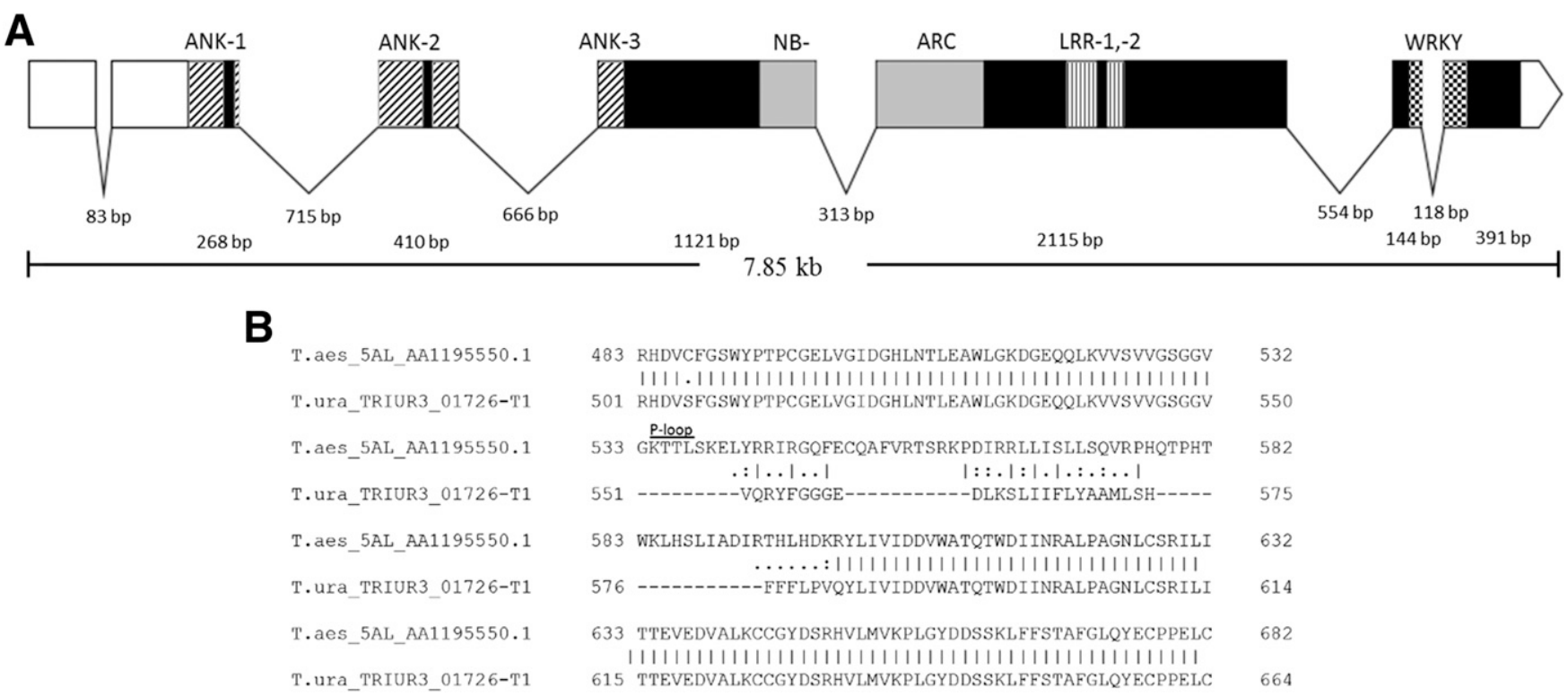

532

550

582

575

632

614

682

664

Fig. 1. Structure of $5 A L-B 4$. A, $5 A L-B 4$ encodes seven exons and six introns with the predicted untranslated regions (empty boxes), ankyrin, NB-ARC, LRR, and WRKY domains indicated. B, Sequence alignment between $5 A L-B 4$ and ancestral copy in Triticum urartu, indicating sequence dissimilarity around the P loop. 
establish a standard curve for reverse transcription quantitative PCR analysis not consistent. The low levels of NLR expression is known and hinders expression analysis (Du Preez et al. 2008; Fossdal et al. 2012). Variation was detected in both NILs for the expression of $5 A L-B 4$ from nine timepoints (Fig. 2). In the susceptible Tugela, only significant $(P=0.023)$ downregulation was detected from the early to late timepoints. Downregulation occurred for 0 to $4 \mathrm{~h}$ postinoculation (hpi) $(P=0.031), 0$ to 48 hpi $(P=0.023), 1$ to 48 hpi $(P=0.05), 4$ to 48 hpi $(P=0.05)$, and 6 to 48 hpi $(P=0.049)$. In the resistant Tugela DN, significant upregulation was detected at the early timepoints for 0 to 6 hpi $(P=0.044), 0.5$ to 1 hpi $(P=0.036), 0.5$ to 6 hpi $(P=$ $0.029)$, and 0.5 to 8 hpi $(P=0.036)$. This was followed by significant downregulation of transcript numbers for 0 to $24 \mathrm{hpi}$ $(P=0.035), 1$ to 24 hpi $(P=0.035), 6$ to 24 hpi $(P=0.008), 8$ to 24 hpi $(P=0.033)$, and 1 to 48 hpi $(P=0.05)$.

\section{A Triticum urartu NLR-ID contributed the core of $5 A L-B 4$.}

Phylogenetic analysis of the full-length protein sequences closely related to $5 A L-B 4$ revealed that $T$. urartu TRIUR3_01726 is the closest relative to $5 A L-B 4$ (Fig. 3). T. urartu is the donor of the A genome in hexaploid wheat. The core sequence of TRIUR3_01726 served as a scaffold for the integration of the WRKY domain now present in $5 A L-B 4$ (Fig. 3, solid black arrow). The protogene of $5 A L-B 4$ follows speciation of the pooid, oryzoid, and panicoid lineages across 40 million years, with possible duplication and divergence occurring at each speciation event. A duplication and divergence event is present where the oryzoid species diverge from panicoid and pooid species, as identified by related genes in Oryza species (Fig. 3, open gray arrow at divergence nodes). Soon after, the gene in the panicoid and pooid lineages duplicated, diverged, and entered the pooid clade, with Brachypodium distachyon occurring basal to the divergence between Hordeum and Triticum species (Fig. 3, open gray arrow). The NLR core continued to duplicate and diversify within barley and wheat species, with barley occurring basal to each branch, including the branch containing the $5 A L-B 4$ gene and its homeologs TRIAE_CS42_5B-AA13L_TGACV 1_405227_AA1322580 (5BL-AA13) and TRIAE_CS42_5DAA14L_TGACv1_434466_AA1436530 (5DL-AA14) (Fig. 3, solid gray arrows). Respectively, $5 B L-A A 13$ and $5 D L-A A 14$ have fused with an Apetala 2 (AP2) and a WRKY-WSKY domain independently of the $5 A L-B 4$ WRKY fusion.

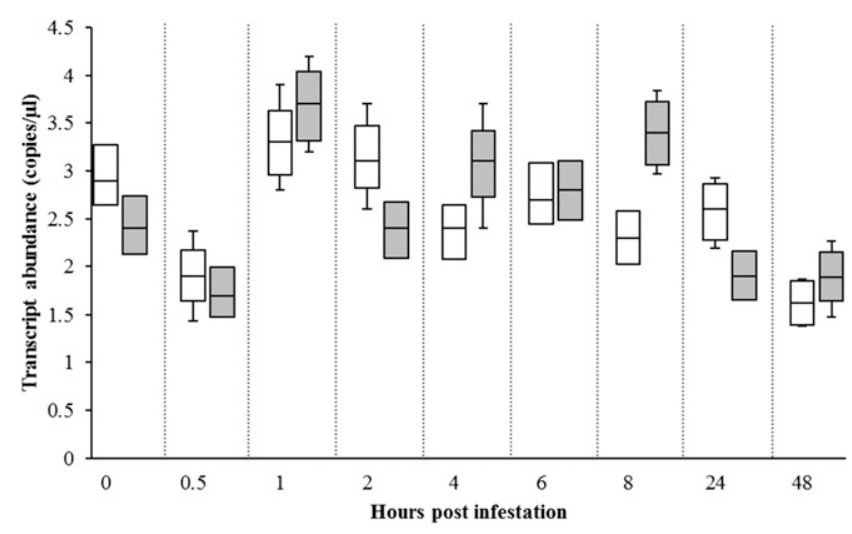

Fig. 2. Differential expression of $5 A L-B 4$ transcripts between Tugela (white bars) and Tugela DN (gray bars) near-isogenic lines (NILs). Significant downregulation of transcript levels in the susceptible NIL Tugela was detected from the early to late timepoints ( 4 to $48 \mathrm{~h}$ postinoculation [hpi], $P \leq$ 0.05). In the resistant NIL Tugela DN, significant upregulation from the early to intermediary ( 0 to $8 \mathrm{hpi}, P \leq 0.05)$ timepoints was detected, followed by significant downregulation at 24 and 48 hpi $(P \leq 0.05)$.
TaWRKY50, the most likely candidate for $5 A L-B 4$ integration.

To further understand the integration of a WRKY domain onto the NLR $5 A L-B 4$, we investigated the phylogenetic placing of the $5 A L-B 4$-specific WRKY domain. All publicly available $T$. aestivum WRKY domain amino acid sequences were aligned to the WRKY and WRKY-WSKY domains on the two homeologs occurring on the 5AL and 5DL chromosomes (Fig. 4). This analysis indicated that the integrated WRKY domain on the $5 D L-A A 14$ (Fig. 4, solid gray arrow) homeolog is a perfect match to TaWRKY41. This integration event conforms to a group I WRKY domain, with two WRKY domains occurring in close proximity of each other. Thus, the core NLR recruited the $5 D L-A A 14$ WRKY domain from the active transcription factor TaWRKY41. Surprisingly, there is no matching TaWRKY domain that corresponds to the integration in $5 A L-B 4$ (Fig. 4, solid black arrow), with the closest match being TaWRKY50 at $79.7 \%$ shared homology across the 69 studied amino acids comprising the domain. The WRKY that was integrated onto $5 A L-B 4$ also originated from group III WRKYs that are characterized by additional amino-acid inclusions and the occurrence of $\mathrm{C} 2 \mathrm{HC}$ zinc finger conformation at the $\mathrm{N}$ terminal end (Eulgem et al. 2000). Interestingly, the two domains on $5 D L$-AA14 contain the two group I WRKY domains with the first being a WRKY motif. However, it also contains the extra amino acids that are present in the group III WRKYs that results in the grouping of the second WSKY motif with group III WRKYs.

\section{Multiple integration events \\ since monocotyledonous divergence.}

It was postulated that the integrated WRKY fusions in monocotyledonous species occurred prior to the divergence between panicoid, pooid, and oryzoid species and involved a WRKY46 homolog (Sarris et al. 2016). Analysis of the integrated WRKY domains from a representative number of monocotyledonous NLR-IDs reveals that integration of the WRKY domain is host- and lineage-specific (Supplementary Fig. S1). Indeed, the various integration events were not based on WRKY46 and involve unrelated WRKY homologs. For most of the integration events, the NLR-ID WRKY did not diverge extensively from the WRKY that it could have originated from. However, in the case of $5 A L-B 4$, this is not evident (Fig. 4). Analysis indicated that the closest WRKY to the one found on $5 A L-B 4$ is TaWRKY50, but with extensive divergence from the original sequence.

\section{The effect of $5 A L-B 4$ transcript silencing on aphid and plant performance.}

The role of $5 A L-B 4$ in the wheat $-D$. noxia interaction was further evaluated using Barley stripe mosaic virus (BSMV)based gene silencing (VIGS). The expression of $5 A L-B 4$ was knocked down in resistant Tugela $\mathrm{DN}$, using a unique genespecific sequence following the WRKY domain. Absolute quantification using ddPCR was used to determine the levels of $5 A L-B 4$ silencing achieved by VIGS compared with transcript levels in the susceptible and resistant controls. BSMV treatment reduced the expression levels of $5 A L-B 4$ by $40 \%$ compared with the uninoculated susceptible and resistant controls (Fig. 5B). Levels of $5 A L-B 4$ were slightly elevated in the TDN+ $\mathrm{BSMV}_{0}$ treatment, most likely due to the stress of viral infection. These levels of silencing were similar across three replications of the VIGS experiments and were similar to those observed in other VIGS studies for non-NLR targets (Schultz et al. 2015; Senthil-Kumar and Mysore 2011).

The reproduction of aphid foundresses in individual clip cages was monitored to determine the effect of silencing $5 A L-B 4$ 
on D. noxia RWASA-1 performance (Table 1; Fig. 5C). The average number of nymphs born per day on TDN+BSMV ${ }_{5 A L-B 4}$ $(n=2.47)$ were similar to the 2.70 nymphs per day on the susceptible Tugela control and were significantly $(P=0.012)$ more than in the resistant controls Tugela DN (1.71 nymphs per day) and $\mathrm{TDN}+\mathrm{BSMV}_{0}$ (1.79 nymphs per day). The average total number of nymphs produced can be used as a measure of aphid fertility (Van Eck et al. 2010). This showed that after

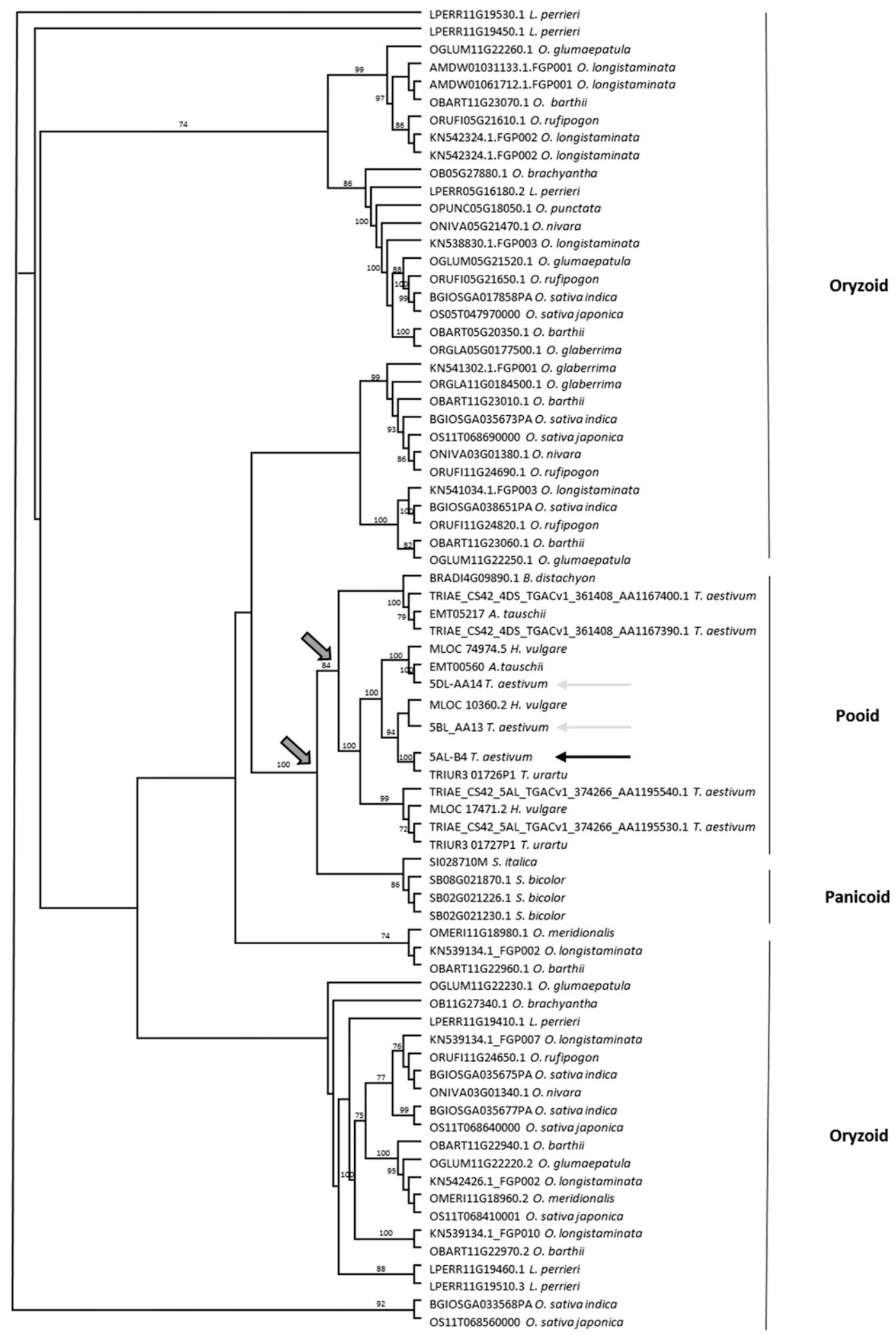

Fig. 3. Phylogenetic analysis indicated that the ANK domain-containing core sequence of Triticum urartu TRIUR3_01726 served as a scaffold for the integration of the WRKY domain of $5 A L-B 4$. The most parsimonious tree is presented with a consistency index of 0.7189 and retention index of 0.7841 . Bootstrap values (1,000 repetitions) above $70 \%$ are indicated. The solid black arrow indicates $5 A L-B 4$, solid gray arrows indicate the two homeologs $5 B L-A A 13$ and 5DL-AA14. The split between the Triticum, Hordeum, and Oryzae clades are indicated with open gray arrows. 
16 days of feeding (21 days after viral infection), a mean total of 22.3 offspring had been produced on $\mathrm{TDN}+\mathrm{BSMV}_{5 A L-B 4}$ plants with a similar number of offspring (24.3) observed on susceptible Tugela plants. In contrast, the resistant controls
Tugela DN and TDN+BSMV ${ }_{0}$, produced 15.4 and 16.1 mean total offspring, respectively. The intrinsic rate of increase $\left(r_{\mathrm{m}}\right)$ was used as a measure of aphid fecundity for the different treatments. The highest calculated rate of increase was observed

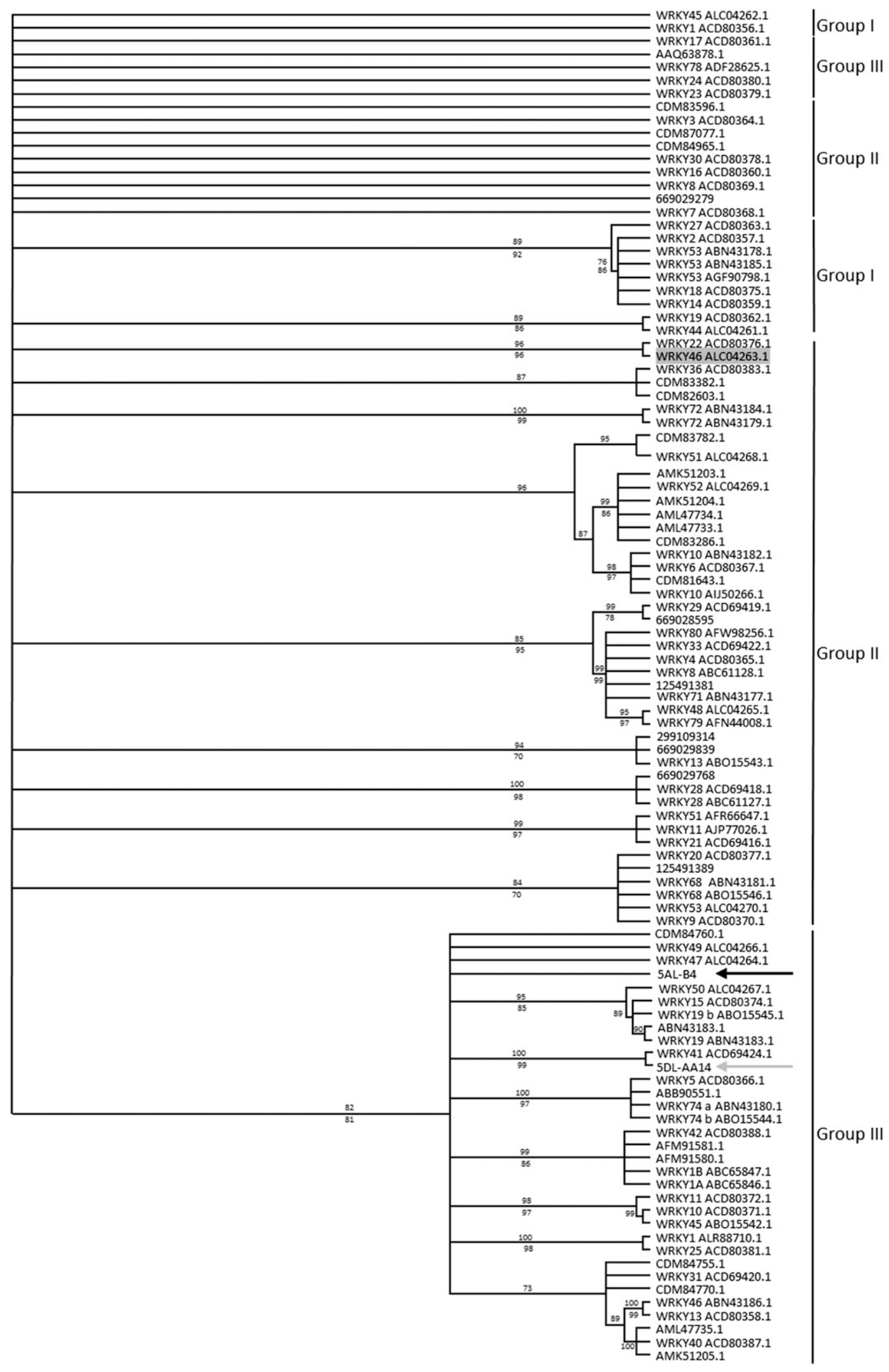

Fig. 4. Triticum aestivum WRKY domain-containing sequences. The most likely candidate WRKY domain that was integrated into $5 A L-B 4$ is TaWRKY50 Multiple WRKY-domain integrations, spanning all the subgroups, are evident in contrast to the proposed ancient integration of AtWRKY46. The most parsimonious tree is presented with a consistency index of 0.5530 and retention index of 0.8530 . Bootstrap values $(1,000$ repetitions, $>70 \%)$ above branches for parsimony analysis and below for maximum likelihood analysis. Best maximum likelihood model fit DCMut+F. Solid black arrow indicates $5 A L-B 4$, solid gray arrow indicate the WRKY-containing homeolog $5 D L-A A 14$. 

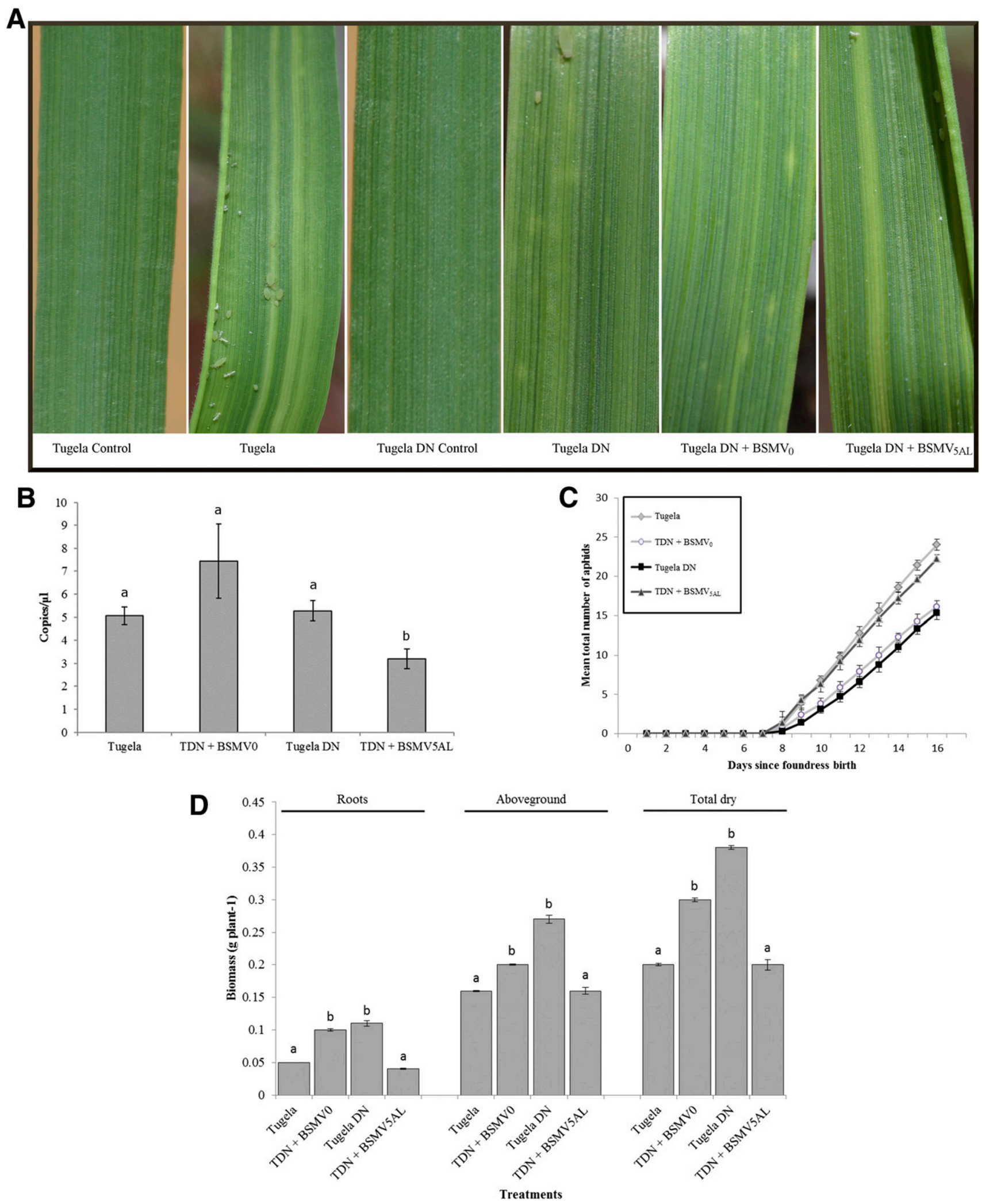

Fig. 5. Plant and aphid phenotypic responses to Barley stripe mosaic virus (BSMV)-mediated virus-induced gene silencing of $5 A L-B 4$. A, Phenotypes of

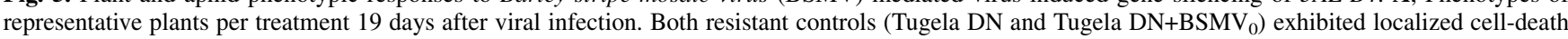
lesions compared with the susceptible (Tugela) and Tugela DN+BSMV ${ }_{5 A L}$-treated plants that exhibited longitudinal chlorotic streaks characteristic of RWASA-1 infestation on susceptible cultivars. Tugela and Tugela DN Control represent uninfested plants and Tugela and Tugela DN infested plants. B, Knockdown of $5 A L-B 4$ measured using droplet digitalpolymerase chain reaction. C, Mean total aphid production of ten plants per treatment over 16 days. Aphids feeding on Tugela and Tugela DN had the highest and lowest mean production of nymphs, respectively, with the number of nymphs produced on silenced plants comparable to those on susceptible plants. D, Individual plant tissues and total dry plant biomass for each treatment following 16 days of aphid feeding on each treatment. Resistant controls had significantly higher biomass for all components compared with the susceptible control and the Tugela DN+ BSMV $_{5 A L}$-treated plants. 
in aphids feeding on susceptible Tugela plants (0.302), and aphids feeding on the resistant control Tugela DN had the lowest rate of increase $(0.252)$. This was not significantly different from the rate of increase on the TDN+BSMV $V_{0}$ control (0.258). Aphids feeding on TDN+BSMV ${ }_{5 A L-B 4}$ had a significantly higher rate of increase (0.295) compared with the Tugela DN and TDN+BSMV $V_{0}$ controls $(P=0.000011)$. The pre-nymphipositional period was recorded for all treatments; however, there was no significant difference in the number of days from the birth of the foundress to the start of her reproduction between the four treatments. The start of reproduction was, on average, seven days after the birth of the foundress, which is consistent with other VIGS studies performed on this interaction (Anderson et al. 2014).

Silencing of $5 A L-B 4$ in Tugela DN induced phenotypic symptoms similar to the susceptible Tugela. The observed localized cell-death lesions were replaced by yellowing and longitudinal chlorotic streaking similar to infested susceptible Tugela (Fig. 5A). This indicated that lower levels of $5 A L-B 4$ resulted in chlorophyll loss similar to when $D$. noxia feeds on susceptible wheat plants. The above- and below-ground plant biomass was monitored to determine the effects of $5 A L-B 4$ silencing on the health of the plant (Fig. 5D). The above- and below-ground and total dry weight of TDN+BSMV $\mathrm{V}_{5 A L-B 4}$ was similar to the susceptible control Tugela and significantly less $(P=0.0011)$ than that of the Tugela DN and TDN+BSMV controls. Silencing of $5 A L-B 4$ in the TDN+BSMV ${ }_{5 A L-B 4}$ plants did not significantly impact mass $(P=0.05)$, as compared with the empty vector control $\mathrm{TDN}+\mathrm{BSMV}_{0}$ in the absence of aphid infestation (Supplementary Table 1). The severe reduction of plant biomass following a compatible infestation with $D$. noxia was consistent with previous reports (Anderson et al. 2014; Mirik et al. 2009). The reduced accumulation of plant biomass in silenced plants together with an increased $D$. noxia reproductive ability correlated to an inability by the plant to initiate an antibiotic defense response characteristic of Dn1mediated resistance.

\section{DISCUSSION}

Here, we show that silencing of $5 A L-B 4$ confers a susceptible phenotype to resistant Tugela DN upon $D$. noxia infestation. This is evident by increased aphid numbers, lowered plant vigor, loss of localized cell death, and increased chlorotic streaking. The resistance mechanism in wheat plants carrying the $D n 1$ resistance gene against $D$. noxia has been phenotypically characterized as antibiosis. This is typified by the host plant reducing aphid fecundity and adult aphid longevity (Botha et al. 2005). The TDN+BSMV ${ }_{5 A L-B 4}$ plants did not show any alteration in the pre-nymphipositional period of the aphids,

Table 1. Aphid fecundity on silenced and control plants ${ }^{\mathrm{a}}$

\begin{tabular}{lccc}
\hline Treatment & $\begin{array}{c}\text { Mean } \\
\text { births/day }\end{array}$ & $\mathbf{r}_{\mathbf{m}}$ & $\begin{array}{c}\text { Prenymphipositional } \\
\text { period (days) }\end{array}$ \\
\hline Tugela & $2.70 \mathrm{a}$ & $0.303+0.021 \mathrm{a}$ & $7.5+0.5 \mathrm{a}$ \\
TDN+BSMV & $1.79 \mathrm{~b}$ & $0.258+0.013 \mathrm{~b}$ & $7.6+0.5 \mathrm{a}$ \\
Tugela DN & $1.71 \mathrm{~b}$ & $0.252+0.012 \mathrm{~b}$ & $7.8+0.4 \mathrm{a}$ \\
TDN+ & $2.47 \mathrm{a}$ & $0.295+0.02 \mathrm{a}$ & $7.4+0.5 \mathrm{a}$ \\
BSMV $_{5 A L-B 4}$ & & & \\
\hline
\end{tabular}

${ }^{a}$ Plants treated with $\mathrm{BSMV}_{5 A L-B 4}$ had a similar mean aphid production rate and aphid fecundity to the susceptible control, and significantly more than the resistant controls. No difference was observed in the prenymphipositional period for each treatment. Different letters per column indicate statistical significance between values listed in that column. $r_{m}=$ intrinsic rate of increase. but an increase in aphid fecundity compared with resistant controls was evident. Diuraphis noxia infestation on susceptible hosts causes the breakdown of chloroplast and cellular membranes, leading to longitudinal chlorotic streaking (Botha et al. 2005). In resistant hosts, infestation leads to the development of small necrotic lesions similar to the localized celldeath response observed in the hypersensitive response (HR) (Botha et al. 2006; van Ooijen et al. 2008). TDN+BSMV $5 A L-B 4$ plants displayed inhibited formation of necrotic lesions around feeding sites and increased yellowing and extensive chlorotic streaking of the leaves. Tugela DN plants are known to produce reactive oxygen species, specifically $\mathrm{H}_{2} \mathrm{O}_{2}$, which activates downstream defense genes and is known to have a strong signaling and defense role during an incompatible interaction in plants with antibiotic resistance (Moloi and van der Westhuizen 2006; Van Eck et al. 2010). In Dn7-containing plants, which also confers an antibiotic effect on the plant, silencing of phenylalanine ammonia-lyase $(P A L)$ and WRKY53 disrupted the production of $\mathrm{H}_{2} \mathrm{O}_{2}$. This led Van Eck et al. (2010) to postulate that PAL and WRKY53 function as part of the defense cascade downstream of $D n 7$. Taken together, these data could indicate that $5 A L-B 4$ functions close to or, possibly, at the molecular recognition of $D$. noxia by plants harboring the $D n 1$ resistance gene.

WRKY integrated fusions are observed across plant lineages and are considered to represent recurrent fusions of the WRKY domain in diverse hosts. However, Sarris et al. (2016) suggested that an ancient fusion event of an AtWRKY46 homolog was integrated into monocotyledonous species prior to the divergence between wheat, sorghum, barley, and foxtail millet. The divergence between panicoid species (such as sorghum) and pooid species (such as wheat and barley) occurred approximately 40 million years ago (Murphy 2011), with the split from oryzoid species occurring earlier, at 50 million years ago (Bossolini et al. 2007). Our phylogenetic analysis of all the known T. aestivum WRKY-containing proteins indicated that the $5 A L$ - $B 4$-integrated WRKY domain is most closely related to TaWRKY50, while the C-terminal WRKY domain present in the homeolog 5DL-AA14 is identical to TaWRKY41. A closer inspection of the integrated WRKY domains in other monocotyledonous species clearly indicated their unrelatedness to AtWRKY46 but that they cluster together within the panicoid, pooid, oryzoid, and dicot lineages. This supports the recurrent integration of different WRKY domains for each lineage rather than a single ancient integration event prior to the divergence of the monocotyledonous species, as previously proposed. The integration of different WRKY domains onto NLR genes could correlate with the NLR function with evolutionary pressure driving diversification of the WRKY domain within each host.

The WRKY integration into the $5 D L-A A 14$ homeolog is nearly identical to the WRKY integration in Aegilops tauschii and Hordeum vulgare, indicating a very well-conserved NLR-ID following their divergence eight million years ago (Middleton et al. 2014). The 5AL-B4 NLR-ID is comparatively much younger, with its conserved ANK-CC-NLR sequence present in $T$. urartu. This precursor to $5 A L-B 4$ was conferred to the hexaploid wheat progenitors during a hybridization event 0.2 to 1.3 million years ago, when $T$. urartu hybridized with an unidentified B genome species to form tetraploid T. dicoccoides (Middleton et al. 2014). The WRKY domain was integrated once the complete hexaploid genome was formed approximately 8,000 to 10,000 years ago (Middleton et al. 2014), as it has no close relatives in any of the progenitor species. Within T. aestivum, it is assumed to have evolved from a TaWRKY50 protein, its closest relative. It remains unclear why the $5 A L-B 4-$ integrated WRKY has diverged to such an extent, compared 
with the highly conserved WRKY domain in its homeolog $5 D L-A A 14$. We cannot rule out that the WRKY transcription factor $5 A L-B 4$ recruited its domain from could since have been lost from the wheat genome. However, this scenario is highly unlikely, as there is no close homolog other than TaWRKY50 to the integrated WRKY on $5 A L-B 4$ in any of the wheat progenitors. This would indicate that the WRKY would have had to be born and died within a very short span of time. Why $5 A L-B 4$ has an evolved WRKY domain that does not closely match a WRKY transcription factor is under further investigation. The function of the NLR core in defense response would seem to have been crucial and, therefore, would have been maintained within this clade. However, there must be an advantageous selective pressure that exists on the core NLR recruiting additional domains, i.e., WRKY, WRKY-WSKY, and the AP2 domains. The recruiting of multiple domains to the core NLR happened independently in the three homeologous genomes of T. aestivum, possibly through convergent evolution to increase the relevant function of each homolog. As these domains all represent transcription factors, it is tempting to speculate that these homeologs might be guarding transcription factors that are targeted by effectors from pathogens and pests.

The role of TaWRKY50 and its nearest homolog in barley (HvWRKY21) is unclear, although some information is available on a close Arabidopsis homolog, AtWRKY41 $(81.7 \%$ homology). AtWRKY41 is a flagellin-induced gene involved in the incompatible interaction between Arabidopsis and the biotrophic pathogen Pseudomonas syringae pv. tomato. Overexpression of AtWRKY41 increases resistance toward Pseudomonas syringae but decreases the resistance toward the necrotrophic pathogen Pectobacterium carotovorum (Higashi et al. 2008). Pseudomonas syringae suppresses AtWRKY41 expression through a type III secretion system effector in compatible interactions (Higashi et al. 2008). Sarris et al. (2016) subsequently found an NLR-ID with an AtWRKY4I domain that interacts with the effector AvrRps4 from Pseudomonas syringae in a yeast-two hybrid screen. Based on the integration of a similar domain onto $5 A L-B 4$, it is conceivable that these two proteins function in a similar manner, and we propose that $5 A L-B 4$ could be functioning in effector trapping and are currently investigating this.

Sarris et al. (2016) found 14 NLR-IDs occurring as double fusions, in which a protein kinase domain was fused with an additional domain, either sequentially or each domain separated by the NLR core. The integration of more than one domain onto the NLR-ID may serve to detect more than a single effector, or perhaps, one of the two domains may have biochemical activity while the second domain simply detects the effector. These proteins appear to have developed from sequential fusion events, as is most probably the case with $5 A L$ $B 4$. The closest relative of $5 A L-B 4$ is an ankyrin repeat domain containing NLR from the wheat A genome progenitor T. urartu. Unlike $5 A L-B 4$, this protein does not contain an integrated WRKY domain and is $94 \%$ homologous to $5 A L-B 4$ if the nonhomologous $\mathrm{C}$ terminals following the leucine-rich repeat are not considered. These two proteins are the only ankyrin repeat domain containing NLRs that have been identified from extensive database searches. This indicates that a unique fusion event occurred in T. urartu that integrated the ankyrin onto the NLR with subsequent donation to the T. aestivum genome. A second fusion event integrated the WRKY domain onto $5 A L-B 4$ to create a unique NLR-ID protein within the plant kingdom. Interestingly, the $\mathrm{P}$ loop in the ancestral form of $5 A L-B 4$ in T. urartu is missing (Fig. 1B). The conserved p-loop motif in the NB-ARC domain regulates nucleotide binding, and mutations within this motif abrogate the ability of the NLR to confer disease resistance or activate the HR (van Ooijen et al. 2008), which indicates that the ancestral gene may not have been functional and subsequent reactivation may have occurred in $5 A L-B 4$. Additionally, the functionality of the ancestral gene may have been lost after its donation to Triticum spp.

The amino-terminal domain of plant NLRs may be involved in both the detection of the pathogen signal and activation of downstream signaling molecules (Collier and Moffett 2009; DeYoung and Innes 2006). While the majority of NLR proteins contain a toll interleukin 1 receptor or CC domain at their $\mathrm{N}$-termini, some proteins have no sequence $\mathrm{N}$ terminal to the NB-ARC domain. A small number of proteins may have a Solanaceae domain or a BED DNA-binding domain replacing or in conjunction with the CC domain (Collier and Moffett 2009). Ankyrin repeat domain-containing proteins constitute one of the largest protein families in all species and plays a role in protein recognition and binding (Mosavi et al. 2004; Vo et al. 2015). Proteins contain one to 33 repeats, although at least two repeats are necessary to assume a folded structure (Mosavi et al. 2004). In plants, ankyrin domain-containing proteins are involved in a wide variety of biological processes, with the majority linked to defense responses (Vo et al. 2015). Observations of ankyrin protein-protein interactions involved in plant defense show that they bind and perceive effectors in the plasma membrane, cytosolic signal transduction, and activation of nuclear defense gene expression, depending on the subcellular localization signal of the ankyrin repeat domain-containing protein (Vo et al. 2015). In the case of $5 A L-B 4$, the ankyrin repeats may mediate intermolecular interactions, in which they bind proteins involved in the defense-gene cascade once the effector has been detected by the WRKY motif and the receptor has been activated. It can also not be ruled out that they are actively recognizing and interacting with effectors themselves.

Sequencing of $5 A L-B 4$ from the resistant and the susceptible lines revealed no SNPs or indels between them or compared with the Chinese Spring cultivar that would account for a resistant genotype. Focusing on the increase in aphid fecundity and loss of the phenotypic hallmarks of resistance in TDN+ $\mathrm{BSMV}_{5 A L-B 4}$ plants, we propose that $5 A L-B 4$ plays a role in the defense response of wheat against $D$. noxia, suggesting an interaction with an unidentified binding partner to form a receptor complex much like previously reported NLR-ID genes. This binding partner either is absent or mutated in the susceptible line compared with the resistant line.

$5 A L-B 4$ contains domains not found in its closest progenitors. This could be the result of unequal crossing over or gene conversion, as seen in other NLR genes that occur as neighbors at the same locus (Loutre et al. 2009). Thus, these paralogs can serve as sources of variation. However, like $\operatorname{Lr} 10$ and $R G A 2$, $5 A L-B 4$ is a single-copy gene that has no closely related NLR genes at its locus, leaving it without access to variation generation due to crossing over with other paralogs. As no other close relative of the WRKY domain, with TaWRKY50 being the closest, was identified in the wheat genome, it is not clear where the WRKY domain originated. It could be a result of gene conversion and allelic recombination between ancient haplotypes or that divergence of the close relatives preceded duplication (Du Preez et al. 2008), and this would be in accordance with loss of genes after genome duplication events as observed in polyploids (Blanc and Wolfe 2004). A counter argument to this would be that the short timespan might not have allowed for this to have occurred.

This is the first report of a WRKY containing NLR-ID protein that functions during a plant-pest interaction. The role of $5 A L-B 4$ in this defense response is intriguing, as it is not the Dn1 gene, but could well be interacting with it directly. The Dnl gene was mapped to a different portion of the genome and Tugela and Chinese Spring do not have D. noxia resistance. 
Furthermore, there are no SNPs evident between the two alleles in Tugela and Tugela DN or between them and Chinese Spring. Thus, we postulate that $5 A L-B 4$ is a NLR-ID protein that is needed to dimerize with $D n 1$ to facilitate resistance in Tugela DN. We propose the name Associated with Dn resistance 1 (Adnrl) to reflect the loss of $D n 1$ resistance once it has been silenced. Additionally, silencing of the WRKY-containing $5 A L$ $B 4$ and WRKY53 (Van Eck et al. 2010) might be indicative that $D$. noxia has developed analogous effectors to phytopathogens that target the evolutionary conserved WRKY transcription factors functioning in innate immunity.

\section{MATERIALS AND METHODS}

\section{Plant and insect growth conditions.}

All experiments were performed using two NILs, Tugela (RWASA1-susceptible) and Tugela DN (PI 137739-Dn1, RWASA1-resistant) that were obtained from the Agricultural Research Council-Small Grain Institute (ARC-SGI, Bethlehem, South Africa). The resistant Tugela DN was created by back-crossing the Dnl-gene from SA1684 into Tugela. This gene has been mapped to the 7DS chromosome in Tugela DN (Bierman 2015). The plants were grown to the two-leaf stage (Zadoks stage 12) under controlled conditions, at $18^{\circ} \mathrm{C}$ with a 12-h photoperiod for approximately 14 days after germination for all experimental procedures. The RWASA1 aphids were obtained from ARC-SGI and were maintained on commercially available susceptible PAN3434 wheat plants (Pannar Seeds, Greytown, South Africa) under controlled conditions at $18^{\circ} \mathrm{C}$ with 12-h photoperiods before use. The three repetitions of BSMV inoculations were performed on 14 plants per treatment, with uninoculated Tugela and Tugela DN used as the susceptible and resistant controls, respectively.

\section{Sequence and phylogenetic analyses.}

TRIAE_CS42_5AL_TGACv1_374266_AA1195550 (5AL$B 4$ ) is an NLR with similar architecture to NLR-ID decoys against pathogen effectors. It shared homology with Pi36, which was identified as a target for differentially regulated miRNA from the study by Nicolis et al. (2017) and identified as a possible role player in the wheat-D. noxia interaction. This, coupled with its unique architecture, prompted us to study its role in the wheat $-D$. noxia interaction. The full length of the gene was amplified from both NILs, using cDNA, and was cloned into pGEM-T Easy (Promega) for sequence analysis. The amplification was performed using $250 \mathrm{nM}$ of each of three primer sets (Set1-F: 5'-CCGGAAATGTTGCCCTTGTG-3' and Set1-R: 5'-CATAGCACGGTCTTCCGCTCTC-3'; Set2-F: 5'-GCCACGTCCACATGCTTCCTAG-3' and Set2-R: 5'GACGAACCTTGTCTGCGAGTG-3'; Set3-F: 5'-TCCTGCAC ACTGCATCACATGG-3' and Set3-R: 5'-ACGCGCTGAC ATCAAATTCG-3') that spanned the length of the gene using KAPA HiFi HotStart (KAPA Biosystems). The sequences for both NILs were generated and aligned to TRIAE_CS42_ 5AL_TGACv1_374266_AA1195550 to identify polymorphisms. Sequences downloaded from Ensemble were aligned using MAFFT and a phylogenetic analysis was performed using maximum parsimony analysis with PAUP* version 4b10 (Swofford 2002). A maximum likelihood analysis was performed using PhyML to ascertain the placing of $5 A L-B 4$, using standard parameters and set to determine the best fit model. Bootstrap support (1,000 replicates) were determined for the tree branching points. The consistency and retention indices were determined for all the datasets. Full-length sequences were used to determine the placing of $5 A L-B 4$ and only the WRKY domains were considered to identify the closest WRKY relative of $5 A L-B 4$.

\section{Expression analyses.}

Confirmation of the differential regulation for $5 A L-B 4$ in the wheat $-D$. noxia interaction was established using ddPCR. The expression for $5 A L-B 4$ was studied at nine timepoints $(0,0.5,1$, 2, 4, 6, 8, 24, and 48 hpi) in both NILs. Each plant was infested with 20 aphids, which were allowed free movement, and noninfested controls were included as reference points for gene expression. RNA was extracted from five wheat plants per treatment for three biological repeats, using the Plant RNeasy Mini extraction kit (Qiagen). The RNA from the five extractions per sample were mixed in equimolar concentrations, and a total of $1 \mu \mathrm{g}$ of RNA was converted into cDNA, using iScript (Bio-Rad), and was used at a 1:19 dilution as template in a ddPCR reaction containing 2× QX200 ddPCR EvaGreen supermix (Bio-Rad) and $200 \mathrm{nM}$ of primer B4-F (5' -TCCTGCACACTGCATCACATGG$\left.3^{\prime}\right)$ and B4-R (5'-GACGAACCTTGTCTGCGAGTG-3'). Reaction droplets were generated in a QX200 Droplet generator (Bio-Rad) using a DG8 cartridge and the PCR was performed on a T100 thermal cycler (Bio-Rad), using a ramp rate of $2^{\circ} \mathrm{C} \mathrm{s}^{-1}$ and enzyme activation step at $95^{\circ} \mathrm{C}$ for $5 \mathrm{~min}$. This was followed by 40 cycles of $95^{\circ} \mathrm{C}$ for $30 \mathrm{~s}$ and $60^{\circ} \mathrm{C}$ for $1 \mathrm{~min}$. A final signal stabilization step of $4^{\circ} \mathrm{C}$ for 5 min followed by $90^{\circ} \mathrm{C}$ for $5 \mathrm{~min}$ was performed. Data acquisition was performed on a QX200 droplet reader, data was analyzed using QuantaSoft software (Bio-Rad), and statistical significance was determined using the student $t$ test $(P \leq 0.05)$.

\section{VIGS of $5 A L-B 4$}

The sequence following the WRKY domain in $5 A L-B 4$ is unique. An across-species and wheat-specific BLASTn revealed no potential silencing of nontarget transcripts. From this unique sequence a 270-bp fragment was amplified from cDNA using the VIGS-F (5'-ACACGTGCTTGGACTCTGTC$\left.3^{\prime}\right)$ and VIGS-R (5'-CGAATTTGATGTCAGCGCGT-3') primers. The fragment was amplified using KAPA HiFi HotStart ReadyMix, was cloned into the pSL038-1 vector, and was verified through sequencing. The construction of the BSMV silencing vector and method of viral inoculation followed the protocol by Scofield et al. (2005). Viral controls included $\mathrm{BSMV}_{0}$, which is derived from the empty pSL038-1 vector, and $\mathrm{BSMV}_{\mathrm{PDS}}$, which included a transcript that targets the phytoene desaturase gene and acts as a visual marker of correct viral reconstitution. Both Tugela and Tugela DN plants were treated with the virus constructs to ascertain the effect of silencing $5 A L-B 4$ on both NILs. Five days after viral inoculation, the plants were mass-infested with $10 \mathrm{D}$. noxia apterous adults. Additionally, a single apterous aphid was placed inside a clip cage attached to the emergent third leaf to determine the fecundity of the aphids feeding on the different silenced plants. The following day, all aphids apart from one new-born nymph were removed from the cage. The remaining new born was regarded as the foundress and nymphs born to this foundress were counted and removed every $24 \mathrm{~h}$ for 14 days. The $r_{\mathrm{m}}$ for each foundress was estimated according to the equation $r_{\mathrm{m}}=$ $\left[0.738 \times \operatorname{In}\left(M_{d}\right)\right] / d$, where $M_{d}$ is the number of nymphs produced in a period equal to the pre-reproductive time (d) (Wyatt and White 1977). To determine the integrity of the antibiosis defense mechanism in Dnl carrying Tugela DN, the effects of aphid feeding on plant biomass accumulation was assessed. Six days after mass aphid infestation (11 days after viral inoculation), aphids were removed from the third leaf of three experimental plants per treatment and the leaf tissue was collected into liquid nitrogen and stored at $-80^{\circ} \mathrm{C}$, prior to extraction. RNA was extracted from each individual leaf sample by homogenization in liquid nitrogen followed by purification with the RNeasy plant mini kit. ddPCR was used for absolute quantification of $5 A L-B 4$ transcript levels in VIGS-treated 
plants as described previously. At 21 days after viral inoculation, all aphids were removed from three plants per treatment and the above-ground plant biomass was separated from the roots. The roots were rinsed and, together with the above-ground plant biomass, were dried for $48 \mathrm{~h}$ at $40^{\circ} \mathrm{C}$, and their weights were determined. Statistical differences were determined using an analysis of variance with Bonferroni correction.

\section{ACKNOWLEDGMENTS}

The authors thank A. Jacobs for assistance with the phylogenetic analyses and critical discussion on the manuscript and Bio-Rad for the use of the QX200 machine to quantify transcript abundance.

\section{LITERATURE CITED}

Anderson, V. A., Haley, S. D., Peairs, F. B., van Eck, L., Leach, J. E., and Lapitan, N. L. 2014. Virus-induced gene silencing suggests $(1,3 ; 1,4)$ $\beta$-glucanase is a susceptibility factor in the compatible Russian wheat aphid-wheat interaction. Mol. Plant-Microbe Interact. 27:913-922.

Ashikawa, I., Hayashi, N., Yamane, H., Kanamori, H., Wu, J., Matsumoto, T., Ono, K., and Yano, M. 2008. Two adjacent nucleotide-binding siteleucine-rich repeat class genes are required to confer Pikm-specific rice blast resistance. Genetics 180:2267-2276.

Bierman, A. 2015. Mapping and survey sequencing of Dn resistance genes in Triticum aestivum L. Ph.D. thesis. University of Stellenbosch, Stellenbosch, South Africa.

Blanc, G., and Wolfe, K. H. 2004. Widespread paleopolyploidy in model plant species inferred from age distributions of duplicate genes. Plant Cell 16:1667-1678

Bos, J. I., Prince, D., Pitino, M., Maffei, M. E., Win, J., and Hogenhout, S. A. 2010. A functional genomics approach identifies candidate effectors from the aphid species Myzus persicae (green peach aphid). PLoS Genet. 6:e1001216.

Bossolini, E., Wicker, T., Knobel, P. A., and Keller, B. 2007. Comparison of orthologous loci from small grass genomes Brachypodium and rice: Implications for wheat genomics and grass genome annotation. Plant J. 49:704-717.

Botha, A.-M., Lacock, L., van Niekerk, C., Matsioloko, M. T., du Preez, F. B., Loots, S., Venter, E., Kunert, K. J., and Cullis, C. A. 2006. Is photosynthetic transcriptional regulation in Triticum aestivum L. cv. 'Tugela DN' a contributing factor for tolerance to Diuraphis noxia (Homoptera: Aphididae)? Plant Cell Rep. 25:41-54.

Botha, A.-M., Li, Y., and Lapitan, N. L. V. 2005. Cereal host interactions with Russian wheat aphid: A review. J. Plant Interact. 1:211-222.

Collier, S. M., and Moffett, P. 2009. NB-LRRs work a "bait and switch" on pathogens. Trends Plant Sci. 14:521-529.

DeYoung, B. J., and Innes, R. W. 2006. Plant NBS-LRR proteins in pathogen sensing and host defense. Nat. Immunol. 7:1243-1249.

Du Preez, F. B., Myburg, A. A., Venter, E., and Botha, A.-M. 2008. Resistance genes in the Triticeae and the dynamics of divergence before duplication. S. Afr. J. Bot. 74:51-64.

Eulgem, T., Rushton, P. J., Robatzek, S., and Somssich, I. E. 2000. The WRKY superfamily of plant transcription factors. Trends Plant Sci. 5: 199-206.

Fossdal, C. G., Yaqoob, N., Krokene, P., Kvaalen, H., Solheim, H., and Yakovlev, I. A. 2012. Local and systemic changes in expression of resistance genes, NB-LRR genes and their putative microRNAs in Norway spruce after wounding and inoculation with the pathogen Ceratocystis polonica. BMC Plant Biol. 12:105.

Higashi, K., Ishiga, Y., Inagaki, Y., Toyoda, K., Shiraishi, T., and Ichinose, Y. 2008. Modulation of defense signal transduction by flagellin-induced WRKY41 transcription factor in Arabidopsis thaliana. Mol. Genet. Genomics 279:303-312.

Jankielsohn, A. 2013. Host associations of Diuraphis noxia (Homoptera: Aphididae) biotypes in South Africa. J. Econ. Entomol. 106:2595-2601.

Jankielsohn, A. 2016. Changes in the Russian wheat aphid (Hemiptera: Aphididae) biotype complex in South Africa. J. Econ. Entomol. 109: 907-912.

Lapitan, N. L., Li, Y. C., Peng, J., and Botha, A.-M. 2007. Fractionated extracts of Russian wheat aphid eliciting defense responses in wheat. J. Econ. Entomol. 100:990-999.

Le Roux, C., Huet, G., Jauneau, A., Camborde, L., Trémousaygue, D., Kraut, A., Zhou, B., Levaillant, M., Adachi, H., Yoshioka, H., Raffaele, S., Berthomé, R., Couté, Y., Parker, J. E., and Deslandes, L. 2015. A receptor pair with an integrated decoy converts pathogen disabling of transcription factors to immunity. Cell 161:1074-1088.

Loutre, C., Wicker, T., Travella, S., Galli, P., Scofield, S., Fahima, T., Feuillet, C., and Keller, B. 2009. Two different CC-NBS-LRR genes are required for $\mathrm{Lr} 10$-mediated leaf rust resistance in tetraploid and hexaploid wheat. Plant J. 60:1043-1054.

Middleton, C. P., Senerchia, N., Stein, N., Akhunov, E. D., Keller, B., Wicker, T., and Kilian, B. 2014. Sequencing of chloroplast genomes from wheat, barley, rye and their relatives provides a detailed insight into the evolution of the Triticeae tribe. PLoS One 9:e85761.

Mirik, M., Ansley, J., Michels, J., and Elliott, N. 2009. Grain and vegetative biomass reduction by the Russian wheat aphid in winter wheat. Southwest Entomol. 34:131-139.

Moloi, M. J., and van der Westhuizen, A. J. 2006. The reactive oxygen species are involved in resistance responses of wheat to the Russian wheat aphid. J. Plant Physiol. 163:1118-1125.

Morrison, W. P., and Peairs, F. B. 1998. Response model concept and economic impact. Pages 1-11 in: A Response Model for an Introduced Pest-The Russian Wheat Aphid. S. S. Quisenberry, and F. B. Peairs, eds Thomas Say Publications in Entomology. Entomological Society of America, Lanham, MD, U.S.A.

Mosavi, L. K., Cammett, T. J., Desrosiers, D. C., and Peng, Z. Y. 2004. The ankyrin repeat as molecular architecture for protein recognition. Protein Sci. 13:1435-1448.

Murphy, D. 2011. Plants, biotechnology and agriculture. CABI, Wallingford, U.K.

Nicolis, V. F., Greyling, S.-M., and Venter, E. 2017. Isolation of earlyresponsive microRNA from Diuraphis noxia (Hemiptera: Aphididae) resistant wheat. J. Econ. Entomol. 110:1298-1306.

Nirmala, J., Drader, T., Chen, X., Steffenson, B., and Kleinhofs, A. 2010. Stem rust spores elicit rapid RPG1 phosphorylation. Mol. Plant-Microbe Interact. 23:1635-1642

Okuyama, Y., Kanzaki, H., Abe, A., Yoshida, K., Tamiru, M., Saitoh, H., Fujibe, T., Matsumura, H., Shenton, M., Galam, D. C., Undan, J., Ito, A., Sone, T., and Terauchi, R. 2011. A multifaceted genomics approach allows the isolation of the rice Pia-blast resistance gene consisting of two adjacent NBS-LRR protein genes. Plant J. 66 : 467-479.

Rodriguez, P. A., and Bos, J. I. 2013. Toward understanding the role of aphid effectors in plant infestation. Mol. Plant-Microbe Interact. 26: 25-30.

Sarris, P. F., Cevik, V., Dagdas, G., Jones, J. D., and Krasileva, K. V. 2016. Comparative analysis of plant immune receptor architectures uncovers host proteins likely targeted by pathogens. BMC Biol. $14: 8$.

Sarris, P. F., Duxbury, Z., Huh, S. U., Ma, Y., Segonzac, C., Sklenar, J., Derbyshire, P., Cevik, V., Rallapalli, G., Saucet, S. B., Wirthmueller, L., Menke, F. L. H., Sohn, K. H., and Jones, J. D. G. 2015. A plant immune receptor detects pathogen effectors that target WRKY transcription factors. Cell 161:1089-1100.

Schultz, T., van Eck, L., and Botha, A.-M. 2015. Phi-class glutathione-Stransferase is involved in Dn1-mediated resistance. Physiol. Plant. 154: $1-12$.

Scofield, S. R., Huang, L., Brandt, A. S., and Gill, B. S. 2005. Development of a virus-induced gene-silencing system for hexaploid wheat and its use in functional analysis of the $\operatorname{Lr} 21$-mediated leaf rust resistance pathway. Plant Physiol. 138:2165-2173.

Senthil-Kumar, M., and Mysore, K. S. 2011. Virus-induced gene silencing can persist for more than 2 years and also be transmitted to progeny seedlings in Nicotiana benthamiana and tomato. Plant Biotechnol. J. 9:797-806.

Sinapidou, E., Williams, K., Nott, L., Bahkt, S., Tör, M., Crute, I., BittnerEddy, P., and Beynon, J. 2004. Two TIR:NB:LRR genes are required to specify resistance to Peronospora parasitica isolate Cala2 in Arabidopsis. Plant J. 38:898-909.

Smith, C. M., Liu, X., Wang, L. J., Liu, X., Chen, M. S., Starkey, S., and Bai, J. 2010. Aphid feeding activates expression of a transcriptome of oxylipin-based defense signals in wheat involved in resistance to herbivory. J. Chem. Ecol. 36:260-276.

Smith, C. M., Schotzko, D. J., Zemetra, R. S., and Souza, E. J. 1992. Categories of resistance in plant introductions of wheat resistant to the Russian wheat aphid (Homoptera: Aphididae). J. Econ. Entomol. 85: 1480-1484.

Swofford, D. L. 2002. Phylogenetic analysis using parsimony (* and other methods). Version 4. Sinauer Associates, Sunderland, MA, U.S.A.

Van Eck, L., Schultz, T., Leach, J. E., Scofield, S. R., Peairs, F. B., Botha, A.-M., and Lapitan, N. L. V. 2010. Virus-induced gene silencing of WRKY53 and an inducible phenylalanine ammonia-lyase in wheat reduces aphid resistance. Plant Biotechnol. J. 8:1023-1032. 
van Ooijen, G., Mayr, G., Kasiem, M. M. A., Albrecht, M., Cornelissen, B. J. C., and Takken, F. L. W. 2008. Structure-function analysis of the NB-ARC domain of plant disease resistance proteins. J. Exp. Bot. 59: 1383-1397.

Vo, K. T. X., Kim, C. Y., Chandran, A. K. N., Jung, K. H., An, G., and Jeon, J. S. 2015. Molecular insights into the function of ankyrin proteins in plants. J. Plant Biol. 58:271-284.

Wang, X., Richards, J., Gross, T., Druka, A., Kleinhofs, A., Steffenson, B. Acevedo, M., and Brueggeman, R. 2013. The rpg4-mediated resistance to wheat stem rust (Puccinia graminis) in barley (Hordeum vulgare) requires Rpg5, a second NBS-LRR gene, and an actin depolymerization factor. Mol. Plant-Microbe Interact. 26:407-418.

Will, T., Tjallingii, W. F., Thönnessen, A., and van Bel, A. J. E. 2007. Molecular sabotage of plant defense by aphid saliva. Proc. Natl. Acad. Sci. U.S.A. 104:10536-10541.

Wyatt, I. J., and White, P. F. 1977. Simple estimation of intrinsic increase rates for aphids and tetranychid mites. J. Appl. Ecol. 14: 757-766 